BLADDER CANCER

\title{
Deep learning in MIBC
}

A deep learning algorithm has demonstrated comparable or superior performance to pathology experts in predicting muscle-invasive bladder cancer (MIBC) molecular subtypes from histopathological slides, according to a new study.

Slides from two cohorts of patients with chemotherapynaive MIBC were used for training and validation of the algorithm. The first comprised 407 patients from The Cancer Genome Atlas (TCGA) bladder cancer cohort, and the second consisted of 16 patients from University Clinic Hospital Erlangen. Training and evaluation of performance metrics were carried out using 363 TCGA slides, and further validation testing used 16 slides from the second cohort.

The reference subtype for each patient was based on expression of a 21-gene panel. Basal, luminal and luminal p53-like tumours were identified, and tumours negative for identifying genes were classified as double-negative.

Slides were digitized and annotated by a pathologist to identify regions of interest. These regions of interest were divided into $512 \times 512$-pixel tiles, which were indi-

In the validation cohort, AUC across all subtypes was 0.85 vidually analysed and classified by the algorithm. Each patient tumour was assigned a subtype based on the majority of tiles for its respective slide, weighted by the prediction certainty for each tile. In addition, class activation maps (CAMs) were generated for each slide to highlight the histomorphological features used by the algorithm to assign subtypes.

In the TCGA cohort, the algorithm achieved an area under the curve (AUC) across all subtypes of $0.89( \pm 0.03)$ and $0.87( \pm 0.03)$ using microaveraging and macroaveraging, respectively. AUC values were also reported for the individual basal $(0.89 \pm 0.04)$, luminal $(0.88 \pm 0.06)$, luminal $p 53$-like $(0.89 \pm 0.04)$ and double-negative $(0.76 \pm 0.12)$ subtypes. Mean validation accuracy per patient was $69.91 \%$. In the validation cohort, AUC across all subtypes was 0.85 using microaveraging or macroaveraging, and accuracy per patient was $75.00 \%$.

Four pathology experts provided with 186 tiles selected at random from 36 patients achieved an overall accuracy rate of $38.19 \%$. None was able to match the accuracy rate of the algorithm in subtype classification. However, when presented with whole-slide images for the 36 patients, as well as with additional information on histopathological features highlighted by the CAMs for each image, overall accuracy increased to $58.89 \%$, and some pathologists exceeded the algorithm's accuracy rate.

Tim Thomas

ORIGINAL ARTICLE Woerl, A.-C. et al. Deep learning predicts molecular subtype of muscle-invasive bladder cancer from conventional histopathological slides. Eur. Urol. https://doi.org/10.1016/j.eururo.2020.04.023 (2020)

RELATED ARTICLE Goldenberg, S. L. et al. A new era: artificial intelligence and machine learning in prostate cancer. Nat. Rev. Urol. 16, 391-403 (2019)

\section{Targeting DNA repair defects in prostate cancer}

Results from a phase III randomized controlled trial show that olaparib treatment resulted in better progression-free survival (PFS) and other objective outcomes than androgen signalling inhibition in men with metastatic castration-resistant prostate cancer (mCRPC) whose disease progressed during abiraterone or enzalutamide treatment and who had mutations in genes involved in homologous recombination DNA repair (HRR).

Advanced prostate cancer carries several genomic aberrations. For example, DNA repair gene alterations are observed in up to $30 \%$ of mCRPCs, including mutations in genes important in the repair of double-strand DNA breaks, such as the HRR genes BRCA1 and BRCA2 and the DNA damage checkpoint activator ATM. Loss-of-function alterations in these and other genes are associated with aggressive disease. Poly(ADP-ribose) polymerase (PARP) is involved in the repair of single-strand DNA breaks and PARP inhibition can lead to cell death in tumour cells with deficiencies in HRR pathways.

The PROfound trial included men with $\mathrm{mCRPC}$ with alterations in prespecified genes involved in HRR whose disease had progressed during abiraterone or enzalutamide treatment. Patients with $\geq 1$ alteration in BRCA1, BRCA2 or ATM were assigned to cohort $A$ and those with alterations in any of 12 other prespecified genes that are also involved in HRR were assigned to cohort B. Patients were then randomly assigned (2:1) to receive the PARP inhibitor olaparib, or enzalutamide or abiraterone at the physician's discretion. The trial's primary end point was PFS assessed by positive imaging for soft tissue or bone lesion progression, or death. Secondary end points included objective response rate (ORR), time to pain progression and overall survival.
In cohort A, which comprised 162 and 83 patients who received olaparib or control treatment, respectively, the median PFS was significantly longer in the olaparib group than in the control group (7.4 months versus 3.6 months; HR 0.34, 95\% CI 0.25-0.47; $P<0.001)$ at the analysis time point when disease had progressed in $71 \%$ of men. The ORR in men who could be evaluated was $33 \%$ ( 28 of 84 patients) in the olaparib group and $2 \%$ ( 1 of 43 patients) in the control group (OR 20.86, 95\% CI 4.18-379.18; $P<0.001)$. Finally, interim analysis revealed longer median overall survival for olaparib than for the control treatment (18.5 months versus 15.1 months, respectively; HR 0.64, 95\% CI 0.43-0.97; $P=0.02$ ).

\section{interim analysis revealed longer median overall survival for olaparib than for the control treatment}

Overall, in the combined cohorts $A$ and B, PFS was significantly longer in men receiving olaparib than in those receiving control treatment (5.8 months versus 3.5 months; HR $0.49,95 \%$ CI $0.38-0.63 ; P<0.001$ ), the ORR was $22 \%$ versus $4 \%$ and overall survival at interim analysis was 17.5 months versus 14.3 months (HR 0.67, 95\% CI 0.49-0.93), respectively. The incidence of grade $\geq 3$ adverse events was higher for olaparib than for control treatment and one death in each group was thought to relate to trial treatment.

Clemens Thoma

ORIGINAL ARTICLE de Bono, J. et al. Olaparib for metastatic castration-resistant prostate cancer. N. Engl. J. Med. 382, 2091-2102 (2020) RELATED ARTICLES Ku, S.-Y. et al. Towards precision oncology in advanced prostate cancer. Nat. Rev. Urol. 16, 645-654 (2019)|Antonarakis, E. S. Olaparib for DNA repair-deficient prostate cancer - one for all, or all for one? Nat. Rev. Clin. Oncol. https://doi.org/10.1038/s41571-020-0395-x

(2020) 